\title{
Breast metastases from primary lung cancer: a retrospective case series on clinical, ultrasonographic, and immunohistochemical features
}

\author{
Bo Wang ${ }^{1 \#}$, Ying Jiang ${ }^{2 \#}$, Shi Yu Li ${ }^{1}$, Rui Lan Niu ${ }^{1}$, Justin D. Blasberg ${ }^{3}$, Jussuf T. Kaifi ${ }^{4}$, Gang Liu ${ }^{5 *}$, \\ Zhi Li Wang ${ }^{1,2 *}$
}

${ }^{1}$ Department of Ultrasound, The First Medical Center, Chinese People's Liberation Army General Hospital, Beijing, China; ${ }^{2}$ School of Medicine, Nankai University, Tianjin, China; ${ }^{3}$ Section of Thoracic Surgery, Department of Surgery, Yale School of Medicine, New Haven, CT, USA; ${ }^{4}$ Division for Cardiothoracic Surgery, Ellis Fischel Cancer Center, University of Missouri, Columbia, MO, USA; ${ }^{5}$ Department of Radiology, The First Medical Center, Chinese People's Liberation Army General Hospital, Beijing, China

Contributions: (I) Conception and design: ZL Wang, G Liu; (II) Administrative support: ZL Wang; G Liu; (III) Provision of study materials or patients: ZL Wang, G Liu; (IV) Collection and assembly of data: B Wang, Y Jiang; (V) Data analysis and interpretation: B Wang, Y Jiang; (VI) Manuscript writing: All authors; (VII) Final approval of manuscript: All authors.

\#These authors contributed equally to this work as co-first authors.

*These authors contributed equally to this work as co-corresponding authors.

Correspondence to: Zhi Li Wang, MD; Gang Liu, MD. First Medical Center of Chinese People’s Liberation Army General Hospital, 28 Fuxing Road, Beijing 100853, China. Email: wzllg@sina.com; 13611245784@126.com.

Background: Lung cancer metastases to the breast are less common and consequently have received much less attention in clinical practice. The purpose of this study was to provide a better understanding of clinical, ultrasonographic, and immunohistochemical features of breast metastases from primary lung cancer.

Methods: This retrospective case series included patients with breast metastases from primary lung cancer between January 2012 and December 2020. Clinical features, ultrasonographic characteristics, and immunohistochemical findings were evaluated in this analysis.

Results: In all, 7 cases (mean \pm standard deviation age: $57.4 \pm 8.3$ years; range, $49-70$ years) were evaluated. The maximum size of breast lesions in 6 cases ranged from 1.2 to $4.5 \mathrm{~cm}$, while 1 case showed a diffused pattern. Ultrasound features of breast metastases from lung cancer were irregular $(5 / 7,71.4 \%)$, indistinct $(6 / 7,85.7 \%)$, hypoechoic $(7 / 7,100.0 \%)$, and parallel $(6 / 7,85.7 \%)$ masses without calcification. Immunohistochemical staining test was positive for thyroid transcription factor 1 (TTF-1) in all patients $(7 / 7$, $100.0 \%), 3$ cases $(3 / 5,60.0 \%)$ were negative for p63, 5 cases $(5 / 5,100.0 \%)$ were positive for cytokeratin 7 (CK7), 4 cases $(4 / 5,80.0 \%)$ were positive for napsin A.

Conclusions: The ultrasonographic features of lung metastases to the breast are clinically important to understand. A known history of the primary lung cancer is of great importance when evaluating patients with a breast nodule. The presence of an ipsilateral lung cancer, breast nodule and axillary lymphadenopathy should be considered with pathological and immunohistochemical data to differentiate breast metastases from a primary breast malignancy in this setting.

Keywords: Breast metastases; lung cancer; ultrasonography; immunohistochemistry; case series

Submitted Apr 15, 2021. Accepted for publication Jul 19, 2021.

doi: $10.21037 /$ tlcr-21-542

View this article at: https://dx.doi.org/10.21037/tlcr-21-542 


\section{Introduction}

Lung cancer is one of the most prevalent cancers around the world, resulting in almost one-quarter of all cancer related deaths (1). Metastases of lung cancer to bone, liver, lung, and brain are frequently observed and has garnered significant interest as the identification of stage IV disease has treatment and prognostic significance (2). Lung cancer metastasis to the breast are less common and consequently have received much less attention in clinical practice (3). Although lung cancer metastasis to the breast can spread via hematogenous and lymphatic routes, classic teaching is that the breast is relatively spared given the relatively poor blood supply in the fibrous breast tissue (4). No specific risk factors related to the development of breast metastasis from lung cancer have been identified.

In patients with a known history of lung cancer, the discovery of breast nodules may result in underutilization of surgery and/or systemic therapy if mis-identification of stage IV disease occurs. There were several reports investigated clinical, or molecular characteristics of breast metastases from extramammary malignancies (5). However, they did not summarize the features of breast ultrasound (US). Breast US is widely utilized in such scenarios to assess the imaging characteristics concerning for malignancy. Although a few case reports have described US features suggestive of breast metastasis versus primary breast malignancy $(6,7)$, breast metastasis from lung cancer still pose a significant diagnostic dilemma in clinical practice. In order to apply the appropriate therapy and avoid unnecessary treatment, there is a need to define specific US features of breast metastases from primary lung cancer. To the best of our knowledge, this is the first report that focus on breast metastases from primary lung cancer and correlate ultrasonographic features with histology to help guide clinical decision making. We present the following article in accordance with the AME Case Series reporting checklist (available at https://dx.doi.org/10.21037/tlcr-21-542).

\section{Methods}

\section{Trial design}

This study is a single-center retrospective case series on clinical, ultrasonographic, and immunohistochemical features of breast metastases from primary lung cancer. The study was conducted in accordance with the Declaration of Helsinki (as revised in 2013). The study was approved by the Ethics Committee of the Chinese People's Liberation
Army General Hospital (No. S2020-354-01), and individual consent for this retrospective analysis was waived.

\section{Patients}

From January 2012 to December 2020, pathologically proven cases of primary lung cancer metastases to the breast were searched in Chinese People's Liberation Army General Hospital. All breast pathology was confirmed by biopsy. Patients were excluded if (I) they did not undergo breast and axillary lymph node (LN) US scans, or (II) if clinical, ultrasonography, pathological information was incomplete.

\section{Ultrasonography}

US images were obtained using a $5-12 \mathrm{MHz}$ linear probe and a VISION Ascendus ultrasound system (Hitachi Aloka Medical, Tokyo, Japan), a Voluson E8 Exp (GE Medical Systems, Zipf, Austria), an Apolio 500 platform (Toshiba, Otawara, Japan), a MyLab Twice ultrasound system (Esaote, Genoa, Italia), an iU22 scanner (Philips Ultrasound, Bothell, WA, USA), and an Aixplorer ultrasound system (SuperSonic Imagine, Aix en Provence, France).

Two independent radiologists with more than 10 years' working experience in breast US retrospectively evaluated the sonography features of the lesions using the American College of Radiology breast imaging reporting and data system (ACR-BIRADS) $5^{\text {th }}$ edition. Nodule features including location, multiplicity, size, shape, margin, orientation, echogenicity, calcification, blood flow signal of the lesions, and axillary LN status were recorded. In addition, associated features, including skin thickening, edema, and architectural distortion were also evaluated by ultrasonography. If two radiologists had different opinions, all disagreements were discussed to the point of consensus. If the patients presented with multiple breast nodules, the largest was evaluated.

\section{Immunobistochemical staining}

All samples were evaluated by a pathologist and based on morphology on HE-staining, panels of immunohistochemistry staining were selected as deemed suitable in each case. The data on immunohistochemical staining was also collected retrospectively. These indicators included thyroid transcription factor 1 (TTF-1), creatine kinase (CK), p63, cytokeratin 7 (CK7), napsin A, CK5, synaptophysin (Syn), estrogen receptor (ER), progesterone receptor (PR), human epithelial 
Table 1 Clinical features

\begin{tabular}{|c|c|c|c|c|c|c|c|}
\hline $\begin{array}{l}\text { Patients } \\
\text { No. }\end{array}$ & Age & Sex & Interval & Pathology & Location & Metastases & Symptom \\
\hline 1 & 70 & Female & NA & Adenocarcinoma & Left & Spine, brain, muscle, breast & Palpable breast lesion, pain \\
\hline 2 & 52 & Female & 33 months & Adenocarcinoma & Left & $\begin{array}{l}\text { Supraclavicular, cervical and } \\
\text { axillary lymph nodes, Lung, } \\
\text { spine, breast }\end{array}$ & $\begin{array}{l}\text { Skin edema, palpable breast } \\
\text { lesions }\end{array}$ \\
\hline 4 & 53 & Female & 4 months & Small cell lung cancer & Right & $\begin{array}{l}\text { Lung, brain, axillary and } \\
\text { mediastinal lymph nodes, } \\
\text { breast }\end{array}$ & No symptoms \\
\hline 5 & 53 & Female & $\begin{array}{l}47 \text { months } \\
13 \text { days }\end{array}$ & Adenocarcinoma & Right & $\begin{array}{l}\text { Scapula, rib, brain, axillary } \\
\text { lymph nodes, breast }\end{array}$ & $\begin{array}{c}\text { Skin edema, palpable breast } \\
\text { lesions }\end{array}$ \\
\hline 7 & 68 & Female & $\begin{array}{l}38 \text { months } \\
14 \text { days }\end{array}$ & Large cell lung cancer & Right & $\begin{array}{l}\text { Cervical and retroperitoneal } \\
\text { lymph nodes, brain, scapula, } \\
\text { breast }\end{array}$ & Skin edema, palpable lesion \\
\hline
\end{tabular}

NA, not available.

receptor 2 (HER-2), Ki-67, and others.

\section{Statistical analysis}

Statistical analyses were performed with Microsoft Excel 2019 and IBM SPSS Statistics 21. The continuous data was presented as mean with standard deviation and range. The categorical data was presented as numbers with proportion (\%). The interval of diagnosis of breast metastases was defined as the interval between the date of the detection of primary lung cancer and the date of detection of breast metastases.

\section{Results}

\section{Clinical features}

From January 2012 to December 2020, 13 pathologically proven cases of primary lung cancer metastases to the breast were identified in our institution. Six cases were excluded due to incomplete clinical or ultrasonographic information. In total, 6 females and 1 male (mean \pm standard deviation age: $57.4 \pm 8.3$ years; range, $49-70$ years) were enrolled. The clinical features of 7 cases are shown in Table 1. In this study group, there were 5 cases of lung adenocarcinoma, 1 case with large cell lung cancer, and 1 case with small cell lung cancer. The primary lung cancer was found in the right lung in $4(4 / 7,57.1 \%)$ cases and on the left in $3(3 / 7,42.9 \%)$ cases. In 5 patients, breast metastases were identified between 4 and 47 months after a diagnosis of lung cancer. These 5 cases received at least one modality of therapy, including surgery, chemotherapy, radiotherapy, and/or immunotherapy. In the remaining 2 patients, breast metastases were discovered before the diagnosis of primary lung cancer. All 7 patients had other distant metastases aside from those in the breast, including the brain, spine, rib, scapula, and cervical LNs.

On physical exam, 4 cases $(4 / 7,57.1 \%)$ had breast manifestations of skin edema and a palpable lesion, 1 case $(1 / 7,14.3 \%)$ presented with a palpable breast lesion and pain, 1 case $(1 / 7,14.3 \%)$ presented with only a palpable lesion, and the remaining was asymptomatic. None of the 7 cases showed other clinical symptoms associated with breast malignancies, such as orange peel-like skin changes, nipple discharge, or nipple depression.

\section{US features}

The US features of the 7 included patients are shown in Table 2. All cases showed single-side breast metastases. Among the 7 cases, $6(6 / 7,85.7 \%)$ had ipsilateral breast 


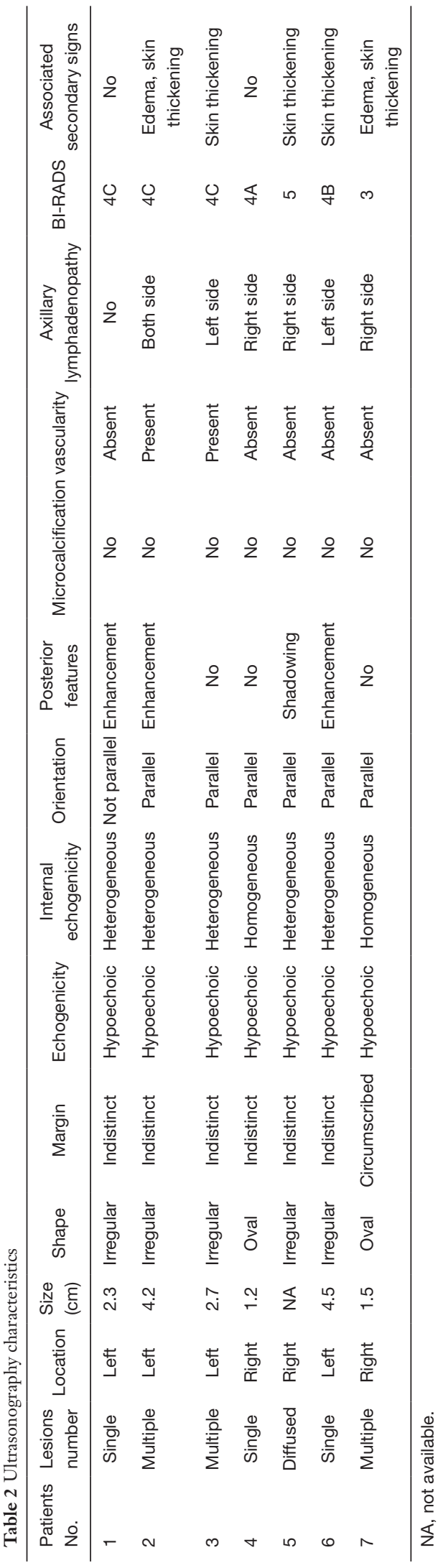

metastases, $1(1 / 7,14.3 \%)$ case had contralateral breast metastasis, $3(3 / 7,42.9 \%)$ cases showed single breast lesions, while the other $4(4 / 7,57.1 \%)$ cases had multiple or diffused lesions. Metastatic lesions were found on the right breast in $3(3 / 7,42.9 \%)$ cases and on the left breast in $4(4 / 7,57.1 \%)$ cases. Additionally, in $5(5 / 7,71.4 \%)$ cases, metastases concurrently were found in axillary LN's on the same side, bilateral axillary LN's in $1(1 / 7,14.3 \%)$ case, and in $1(1 / 7,14.3 \%)$ case presented with no axillary lymphadenopathy was identified.

In our study, the maximum size of breast lesions in 6 cases ranged from 1.2 to $4.5 \mathrm{~cm}$, and 1 case with a diffused breast lesion was identified (Figure 1). The shape of breast metastases in $5(5 / 7,71.4 \%)$ cases was irregular, with the other $2(2 / 7,28.6 \%)$ cases having an oval shape (Figure 2). The margin of metastases in $6(6 / 7,85.7 \%)$ cases was indistinct, while in the remaining case the margin was noted to be well circumscribed. The lesions were hypoechoic in all cases $(7 / 7,100.0 \%)$ and had heterogeneous internal echogenicity in $5(5 / 7,71.4 \%)$ cases, while homogeneous internal echogenicity was found in $2(28.6 \%)$ cases. The lesion orientation of $6(6 / 7,85.7 \%)$ cases was parallel (wider than tall), $3(3 / 7,42.9 \%)$ cases showed enhancement posterior feature (Figure 3), and $1(1 / 7,14.3 \%)$ case had a feature of posterior acoustic shadowing. Microcalcification was not observed in any patient. Color Doppler US detected blood flow in $2(2 / 7,28.6 \%)$ cases. Associated secondary signs, including edema and skin thickening, were found in $5(5 / 7,71.4 \%)$ cases. The final BI-RADS assessment categories were BI-RADS 3 in $1(1 / 7,14.3 \%)$ case, BI-RADS $4 \mathrm{~A}$ in $1(1 / 7,14.3 \%)$ case, BI-RADS $4 \mathrm{~B}$ in 1 $(1 / 7,14.3 \%)$ case, BI-RADS $4 \mathrm{C}$ in $3(3 / 7,42.9 \%)$ cases, BIRADS 5 in $1(1 / 7,14.3 \%)$ case.

\section{Immunohistochemical features}

Immunohistochemical staining test results from 7 cases were collected. All 7 (7/7, 100.0\%) cases were TTF-1 positive; 5 cases underwent p63 staining, among which $2(2 / 5,40.0 \%)$ cases were p63 partially positive and $3(3 / 5$, $60.0 \%)$ were p63 negative; 5 cases received CK7 and napsin A staining, among which $5(5 / 5,100.0 \%)$ cases were CK7 positive, 4 (4/5, 80.0\%) were napsin A positive (Figure 4). Other staining indicators are shown in Table 3.

\section{Discussion}

Lung cancer metastasis to the breast is an extremely rare 

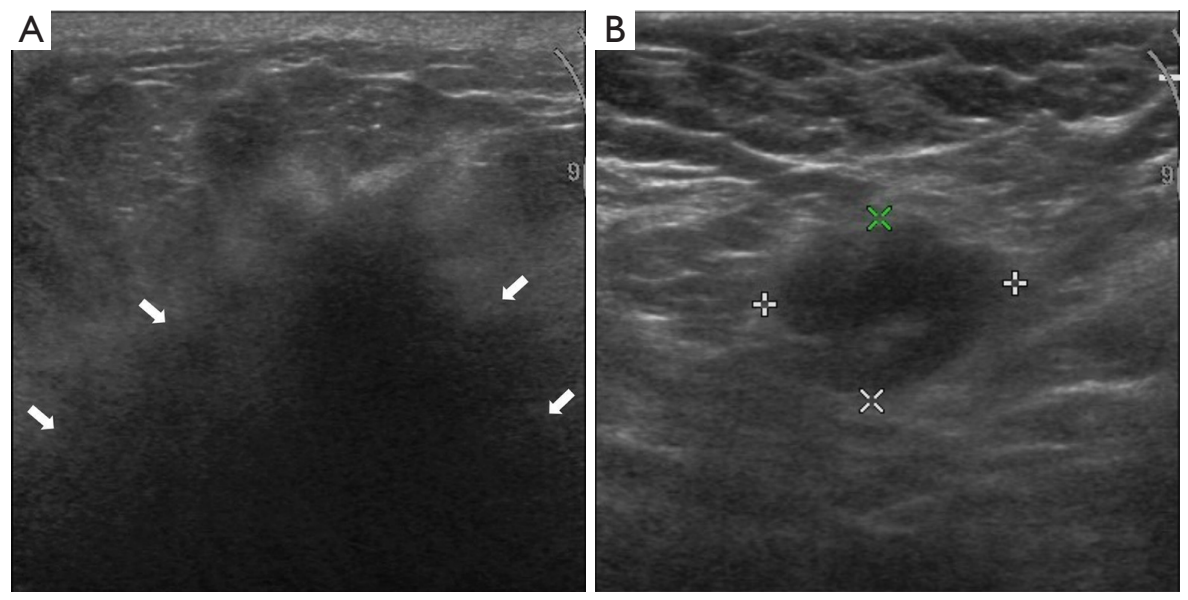

Figure 1 Ultrasonography for breast metastases from primary lung cancer. Patient No. 5: a 53-year-old female with adenocarcinoma of right lung. Ultrasonography shows a diffused irregular indistinct and heterogeneous hypoechoic mass in the right breast with the long axis parallel to the skin, shadowing posterior features (white arrow) (A), and ipsilateral axillary lymph adenopathy (cross symbol) (B).
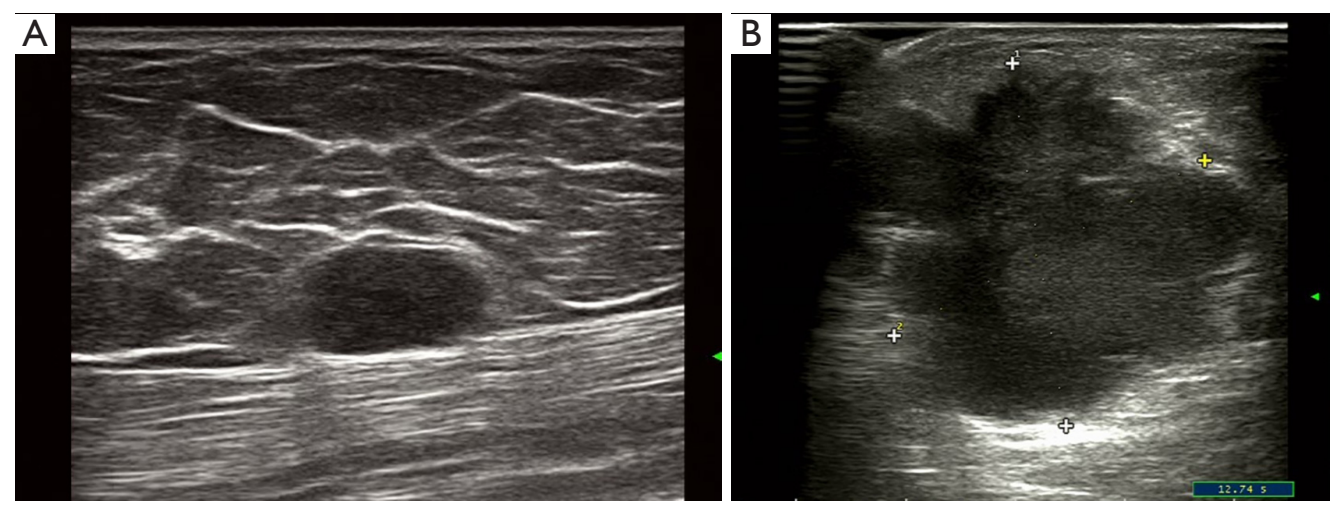

Figure 2 Ultrasonography for breast metastases from primary lung cancer. Patient No. 4: a 53-year-old female with small cell lung cancer of right lung. Ultrasonography shows an oval indistinct and homogeneous hypoechoic mass in the right breast with the long axis parallel to the skin (A), and ipsilateral axillary lymph adenopathy (cross symbol) (B).

clinical presentation with an incidence of just $0.2-1.3 \%(8)$. On the contrary, $21-32 \%$ of breast cancer patients with distant metastases have lesions in the lung (9). In lung cancer cases where a concurrent breast nodule is identified, one should include metastatic lung cancer within the differential. However, some patients may present with a breast nodule as the first clinical manifestation of metastatic lung cancer, confusing the clinical workup of these patients. Under these circumstances, comprehensive understanding both pathologic features associated with lung cancer metastasis and ultrasonography features of nodules that are metastatic to the breast is particularly important.

Selection of appropriate therapy depends on accurate diagnosis of the breast lesions, and is significant for the effective management and life quality of the patients. Patients with primary breast cancer and breast metastasis from lung cancer have different therapy sections and clinical consequences. It has been documented that patients with late-stage lung cancer have a very poor prognosis, while patients with primary breast cancer present with relatively good prognosis $(1,10)$. In clinical practice, it is very challenging to distinguish breast metastasis from primary breast cancer, as metastatic cancer can mimic malignant breast lesions or even benign lesions. The symptoms of orange peel-like skin changes, nipple discharge, or depression are uncommon in breast metastasis (11). In 

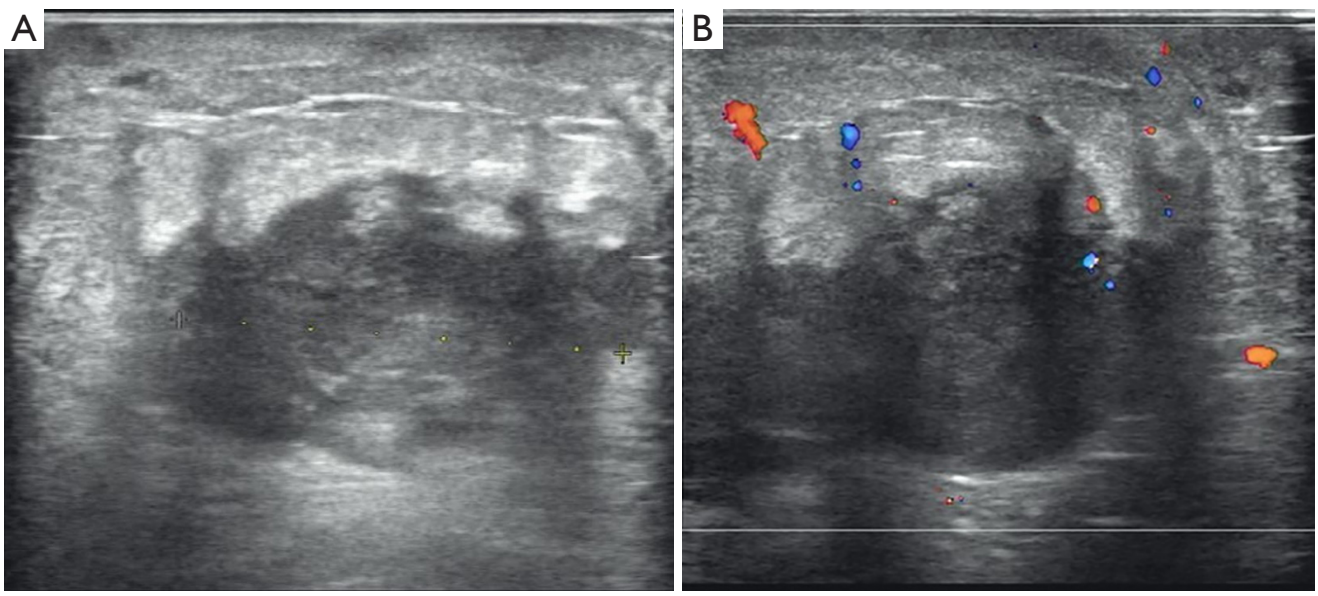

Figure 3 Ultrasonography for breast metastases from primary lung cancer. Patient No. 2: a 52-year-old female with adenocarcinoma of left lung. (A) Ultrasonography shows an irregular indistinct and heterogeneous hypoechoic mass in the left breast with the long axis parallel to the skin, enhancement posterior features (cross symbol); (B) color Doppler flowing imaging shows blood flow signals inside the mass.
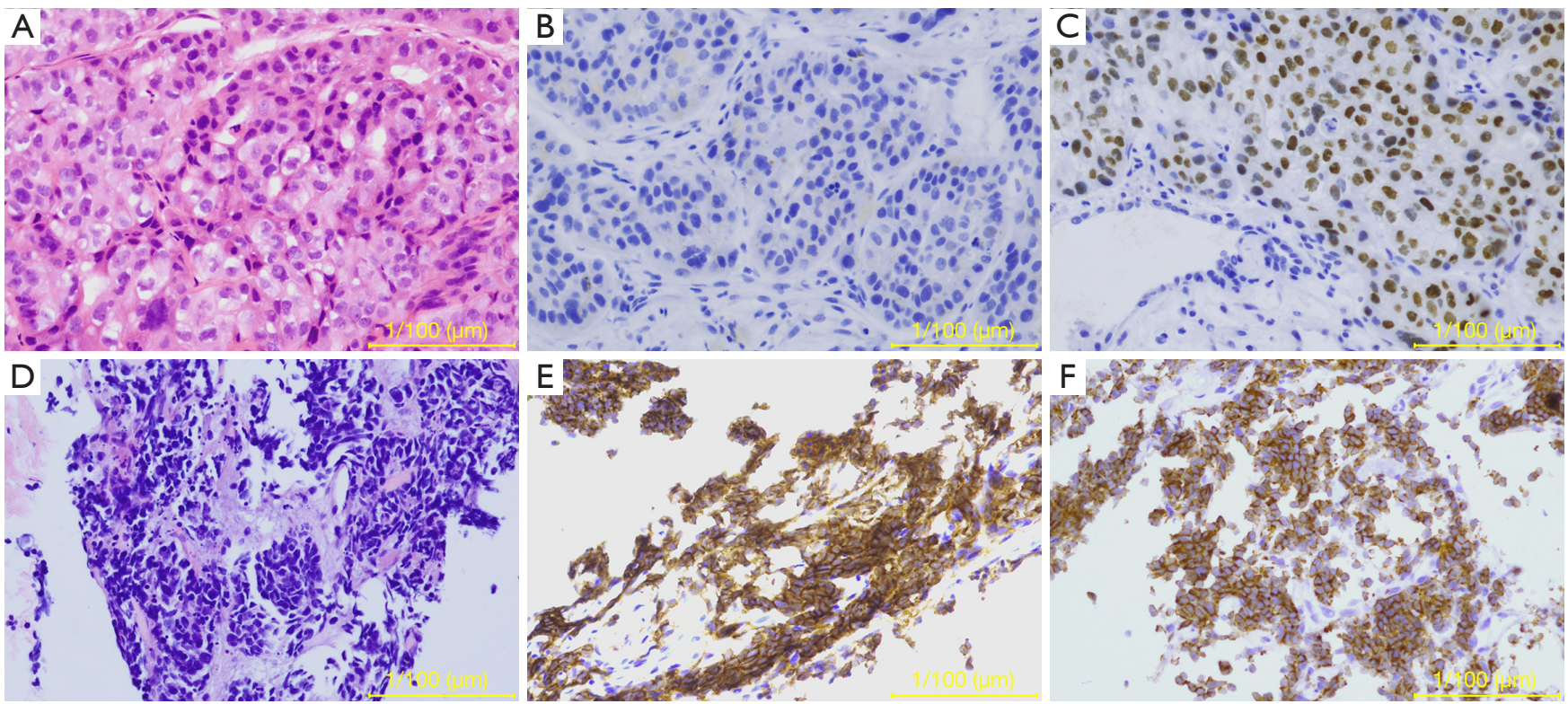

Figure 4 Histological features of breast metastases from primary lung cancer. Patient No. 1: metastasis from lung adenocarcinoma (A, H\&E 200x), napsin A (B, immunostain 200x) and thyroid transcription factor 1 (C, immunostain 200x); Patient No. 4: metastasis from small cell lung cancer (D, H\&E 200x), synaptophysin (E, Immunostain 200x) and cluster of differentiation 56 (F, immunostain 200x). The scale bar is $100 \mu \mathrm{m}$ for A-F.

our study, the most common symptoms were skin edema and painless breast lesions. Edema of the skin may be due to the rapid growth of metastatic cancer, which could be misdiagnosed as inflammatory breast carcinoma (12).

Both single and multiple lesions of unilateral breast were found in our study. The ipsilateral breast metastasis from lung cancer was more prevalent than contralateral metastasis in this study. One study has reported metastasis to bilateral breasts (13). In addition, most of the patients in our study had ipsilateral axillary lymphadenopathy. This is similar to other studies, which report cases of lung cancer with ipsilateral breast metastasis (14-16); metastasis to the contralateral 
Table 3 Immunohistochemistry staining

\begin{tabular}{|c|c|c|c|c|c|}
\hline $\begin{array}{l}\text { Patient } \\
\text { No. }\end{array}$ & TTF-1 & p63 & CK7 & Napsin A & Others \\
\hline 1 & Positive & Partially positive & Positive & Negative & $\begin{array}{c}\text { CK5(-), Syn(-), PR(-), ER(-), HER-2(2+), Ki-67(+60\%), GCDFP-15(-), } \\
\text { GATA-3(-), CgA(-), CD56(-) }\end{array}$ \\
\hline 2 & Positive & Partially positive & Positive & Positive & CK5(-), CK(+), CD68(-) \\
\hline 3 & Positive & NA & Positive & Positive & $\begin{array}{c}\text { CK20(-), Ki-67(+20-30\%), EGFR(+), P53(+), ALK(+), D2-40(-), } \\
\text { Calretinin(-/+) }\end{array}$ \\
\hline 4 & Positive & Negative & NA & NA & CK(weakly +), Syn(+), CgA(-), CD56(+), LCA(-), Ki-67(+70\%) \\
\hline 5 & Positive & Negative & Positive & Positive & CK5(-), CK20(-), CEA(+), CR(-), CK6(-) \\
\hline 6 & Positive & NA & Positive & Positive & $\begin{array}{c}\text { CD56(-), GPC-3(-), GCDFP-15(-), Syn(-), CgA(-), PR(-), ER(-), HER- } \\
\text { 2(focally+), Ki-67(+15\%), GATA-3(-) }\end{array}$ \\
\hline
\end{tabular}

NA, not available; TTF, thyroid transcription factor; CK, creatin-kinase; Syn, synaptophysin; CD, cluster of differentiation; ER, estrogen receptor; PR, progesterone receptor; HER, human epithelial receptor: GCDFP, gross cystic disease fluid protein; EGFR, epidermal growth factor receptor; ALK, anaplastic lymphoma kinase; LCA, leucocyte common antigen; GPC, glypican.

breast has also been previously reported (3), but this finding is less common than ipsilateral metastasis. Indeed, only 1 patient in our study had contralateral breast metastasis. Similarly, in Huang et al.'s (17) review of 19 patients with primary lung adenocarcinoma, 3 had contralateral breast metastases, while the remaining 16 patients had ipsilateral metastases. The mechanism of primary lung cancer metastasis to the ipsilateral breast is still unclear. The lymphatic drainage of the mediastinal LNs through the intercostal or supraclavicular lymph vessels may lead to ipsilateral axillary LN metastasis and breast metastasis (18). Another possibility is lymphatic drainage from the chest wall, which may be involved by the lung cancer cells either via direct extension or seeding by a malignant pleural effusion or the parietal pleura (17). The findings of enlarged ipsilateral axillary LNs may represent evidence for these mechanisms. Thus, an ipsilateral lung cancer, breast lesion and axillary lymphadenopathy should raise one's suspicion of a metastatic lung cancer process. A hematogenous route is also another possible way by which extramammary malignancies metastasize to the breast, and may explain why metastasis can also occur in the contralateral breast. Although stage IV lung cancer to the breast is rare, clinicians should carefully examine the axillary LN and breast when evaluating patients with primary lung cancer.

The common US features of primary breast cancer are irregular, not parallel, indistinct, irregular, hypoechoic, homogeneous masses with microcalcification and posterior acoustic shadowing (19). The common US features of breast metastases from lung cancer are nodules that are irregular, indistinct, hypoechoic, and parallel masses without calcification. The maximum size of breast metastases from lung adenocarcinoma was larger than that of large cell and small cell lung cancer. In addition, internal echogenicity was homogeneous in patients with large cell and small cell lung cancer, while it was heterogenous in patients with adenocarcinoma. Most lesions were parallel to the skin. Posterior acoustic enhancement was also observed in our study. This is different from the US feature of primary breast cancer, where posterior acoustic shadowing is most typical (20). However, we still observed 1 patient with a diffused breast metastasis featuring posterior acoustic shadowing, which, to our knowledge, is the first report of these features in breast metastases. Calcification, which is rarely observed in metastasis to the breast, may be helpful in distinguishing stage IV disease from a primary breast cancer. Only 1 prior study has reported a case of calcifications in lung cancer metastatic to the breast (13). Color blood flow signal was detected in 2 patients, which is different from the abundant vascularity of primary breast carcinoma (21). Sippo et al. (20) and Moreno-Astudillo et al. (22) reported that the US manifestation of other cancer metastases to the breast were round or oval hypoechoic lesions, which could be diagnosed as benign nodules. This study also observed a case of metastatic small cell lung cancer to the breast, which demonstrated an oval shape and indistinct margin, and homogeneous internal echogenicity. In contrast, Luh et al. (23) reported 2 small lung cancer masses with a distinct 
margin and heterogeneous internal echogenicity.

All lesions in this study could be easily identified in US imaging. However, Mun et al. (4) also reported a case of breast metastasis from lung cancer manifesting as nonmass-like lesion with segmental and ductal distribution of multiple small hypoechoic masses and axillary LN enlargement, which could be easily confused with ductal carcinoma in situ or papillary lesions. However, only 1 study has thus far reported non-mass-like breast metastatic lesions from lung cancer in US.

As mentioned above, the differentiation between primary cancer and breast metastases from lung cancer poses a diagnostic dilemma. Immunohistochemical staining is essential in patients with breast nodules. In general, no single marker can reach $100 \%$ specificity or sensitivity, so confirmation through a set of antibodies is essential (24). Choosing a panel for immunohistochemical staining depends on the pathologist assessment of morphology and knowledge of clinical features including earlier malignancy. Some of the antibodies chosen is used to broadly guide when differentiate origin of tumor cells, e.g., CK7 and CK20 can be used to differentiate between origin from lower gastrointestinal tract from, e.g., lung and breast, but not to differentiate between lung and breast (8). Others are chosen to confirm more specific organs and a commonly used panel for lung tumors include TTF-1 and napsin A. TTF-1 is a common marker for the identification of the origin of metastatic mass from lung cancer (25). It is expressed in $70 \%$ to $80 \%$ of small cell carcinomas and lung adenocarcinomas, and is rarely expressed in other types of lung cancer (26). Napsin A, which is positive in $80-90 \%$ of lung adenocarcinomas, is typically reported to be negative in breast cancer $(27,28)$, napsin $\mathrm{A}$ is an alternative biomarker for the diagnosis of lung adenocarcinoma. The combination of TTF-1 and napsin A can provide maximum benefit in clinical practice. In the cases presented in this study, differentiation between origin of tumor in either lung or breast is crucial and common markers for breast cancer include PR, ER, HER-2 and GATA-3. PR, ER and HER-2 are usually negative in breast metastases from lung cancer (29). It is reported that HER2 mutations are identified in about $2 \%$ of NSCLCs and $2 \%$ to $6 \%$ of lung adenocarcinomas $(30,31)$. In this study, we also identified that 2 cases with primary lung adenocarcinoma had positive HER-2 staining, and 1 case with large cell lung cancer had focally positive HER-2 staining. In our study, 2 of 5 cases had a partially positive p63 staining, while the other 3 cases were p63 negative. According to the study of Provenzano et al. (32), 97\% of lung squamous cell carcinomas show a high expression level of p63, while only $7 \%$ of lung adenocarcinomas show a high expression level of p63. Thus, p63 is a helpful indicator to distinguish between squamous cell carcinoma and adenocarcinoma $(33,34)$. p63 positive has been reported to be present in $94-100 \%$ of squamous cell carcinomas with diffused staining in most cases, while 9-32\% of lung adenocarcinomas tend to show focal staining (32). Moreover, focal p63-positive staining can be seen in about $22 \%$ of triple-negative breast cancer cases (35-37). As most breast metastases are from lung adenocarcinoma, p63 may be of limited use in the differentiation of primary and secondary breast cancer. Therefore, in cases with atypical clinical and US features, or in cases without a known primary cancer history, a group of markers is recommended to provide optimal diagnostic accuracy.

This retrospective case series summarized the features of breast metastases from lung cancer by reviewing cases in our center. There are, however, a few limitations to this study. First, the sample size was small and all from one single institution. Second, we did not include elastography or contrast-enhanced US in this study. Thus, we will endeavor to further investigate these issues in future study using cases from multiple centers and a greater variety of techniques. Additionally, this study was not powered to statistically define associations between imaging or pathologic features with risk for metastatic lung cancer, such that these results are more consistent with observations rather than direct correlations.

\section{Conclusions}

Form this observational, retrospective case series, we analyzed the clinical, ultrasonographic, and immunohistochemical features of breast metastases from lung cancer. We conclude that the common US features of breast metastases from lung cancer are nodules that are irregular, indistinct, hypoechoic, and parallel masses without calcification. We believe that these features can help guide clinical decisionmaking including staging and treatment options. A known history of the primary lung cancer is also of great importance when evaluating a patient with breast nodules suspicious for primary versus metastatic disease. The presence of an ipsilateral lung cancer, breast lesion and axillary lymphadenopathy should prompt the clinician to including metastatic lung cancer within the differential diagnosis.

\section{Acknowledgments}

The authors appreciate the academic support from AME 
Lung Cancer Collaborative Group and Dr. Ida Skovgaard Christiansen of Zealand University Hospital.

Funding: This work was supported by the National Natural Science Foundation of China (No. 81771832 and 82071925) and the Military Top Project of Youth Training for Medical Science and Technology (No. 19QNP071).

\section{Footnote}

Reporting Checklist: The authors have completed the AME Case Series reporting checklist. Available at https://dx.doi. org/10.21037/tlcr-21-542

Data Sharing Statement: Available at https://dx.doi. org/10.21037/tlcr-21-542

Conflicts of Interest: All authors have completed the ICMJE uniform disclosure form (available at https://dx.doi. org/10.21037/tlcr-21-542). The authors have no conflicts of interest to declare.

Ethical Statement: The authors are accountable for all aspects of the work in ensuring that questions related to the accuracy or integrity of any part of the work are appropriately investigated and resolved. The study was conducted in accordance with the Declaration of Helsinki (as revised in 2013). The study was approved by the Ethics Committee of the Chinese People's Liberation Army General Hospital (No. S2020-354-01), and individual consent for this retrospective analysis was waived.

Open Access Statement: This is an Open Access article distributed in accordance with the Creative Commons Attribution-NonCommercial-NoDerivs 4.0 International License (CC BY-NC-ND 4.0), which permits the noncommercial replication and distribution of the article with the strict proviso that no changes or edits are made and the original work is properly cited (including links to both the formal publication through the relevant DOI and the license). See: https://creativecommons.org/licenses/by-nc-nd/4.0/.

\section{References}

1. Siegel RL, Miller KD, Jemal A. Cancer statistics, 2020. CA Cancer J Clin 2020;70:7-30.

2. Riihimäki M, Hemminki A, Fallah M, et al. Metastatic sites and survival in lung cancer. Lung Cancer 2014;86:78-84.
3. Ji FF, Gao P, Wang JG, et al. Contralateral breast metastasis from pulmonary adenocarcinoma: two cases report and literature review. J Thorac Dis 2012;4:384-9.

4. Mun SH, Ko EY, Han BK, et al. Breast metastases from extramammary malignancies: typical and atypical ultrasound features. Korean J Radiol 2014;15:20-8.

5. Zhu Y, Liu WW, Wu Q, et al. Clinical and molecular characteristics of secondary breast metastases from primary lung cancer: a study of 22 Chinese cases. Int J Clin Exp Pathol 2020;13:1880-5.

6. Lee SH, Park JM, Kook SH, et al. Metastatic tumors to the breast: mammographic and ultrasonographic findings. J Ultrasound Med 2000;19:257-62.

7. Ko K, Ro JY, Hong EK, et al. Micropapillary lung cancer with breast metastasis simulating primary breast cancer due to architectural distortion on images. Korean J Radiol 2012;13:249-53.

8. Lee AH. The histological diagnosis of metastases to the breast from extramammary malignancies. J Clin Pathol 2007;60:1333-41.

9. Medeiros B, Allan AL. Molecular Mechanisms of Breast Cancer Metastasis to the Lung: Clinical and Experimental Perspectives. Int J Mol Sci 2019;20:2272.

10. Wang T, Nelson RA, Bogardus A, et al. Five-year lung cancer survival: which advanced stage nonsmall cell lung cancer patients attain long-term survival? Cancer 2010;116:1518-25.

11. Lee JH, Kim SH, Kang BJ, et al. Metastases to the breast from extramammary malignancies-sonographic features. J Clin Ultrasound 2011;39:248-55.

12. Malek D, Buccheri S, Dey CB, et al. Lung cancer metastasis to the breast mimicking inflammatory breast carcinoma on imaging. Radiol Case Rep 2019;14:1500-5.

13. Wan X, Zhang H, Zhang Y, et al. Metastases to the Breast from Extramammary Nonhematological Malignancies: Case Series. Int J Gen Med 2020;13:1105-14.

14. Yoon MY, Song CS, Seo MH, et al. A case of metachronous metastasis to the breast from non-small cell lung carcinoma. Cancer Res Treat 2010;42:172-5.

15. Maounis N, Chorti M, Legaki S, et al. Metastasis to the breast from an adenocarcinoma of the lung with extensive micropapillary component: a case report and review of the literature. Diagn Pathol 2010;5:82.

16. Li CS, Chen T, Tu HY. Metastases to the breast from adenocarcinoma of lung: Incidentally detected with routine computed tomography of chest. Chinese Journal of Radiology 2011;36:37-40.

17. Huang HC, Hang JF, Wu MH, et al. Lung 
adenocarcinoma with ipsilateral breast metastasis: a simple coincidence? J Thorac Oncol 2013;8:974-9.

18. Barber TW, Hofman MS, Hicks RJ. Breast lymphatic drainage via the pulmonary lymphatic system. Eur J Nucl Med Mol Imaging 2010;37:2203.

19. Guo R, Lu G, Qin B, et al. Ultrasound Imaging Technologies for Breast Cancer Detection and Management: A Review. Ultrasound Med Biol 2018;44:37-70.

20. Sippo DA, Kulkarni K, Carlo PD, et al. Metastatic Disease to the Breast From Extramammary Malignancies: A Multimodality Pictorial Review. Curr Probl Diagn Radiol 2016;45:225-32.

21. Zhu YC, Zu DM, Zhang Y, et al. A comparative study on superb microvascular imaging and conventional ultrasonography in differentiating BI-RADS 4 breast lesions. Oncol Lett 2019;18:3202-10.

22. Moreno-Astudillo L, Villaseñor-Navarro Y, SánchezGoytia V, et al. A Case Series of Breast Metastases from Different Extramammary Malignancies and Their Literature Review. Case Rep Radiol 2019;2019:9454201.

23. Luh SP, Kuo C, Tsao TC. Breast metastasis from small cell lung carcinoma. J Zhejiang Univ Sci B 2008;9:39-43.

24. Bonacho T, Rodrigues F, Liberal J. Immunohistochemistry for diagnosis and prognosis of breast cancer: a review. Biotech Histochem 2020;95:71-91.

25. Ali RH, Taraboanta C, Mohammad T, et al. Metastatic non-small cell lung carcinoma a mimic of primary breast carcinoma-case series and literature review. Virchows Arch 2018;472:771-7.

26. Lee AHS, Hodi Z, Soomro I, et al. Histological clues to the diagnosis of metastasis to the breast from extramammary malignancies. Histopathology 2020;77:303-13.

27. Turner BM, Cagle PT, Sainz IM, et al. Napsin A, a new marker for lung adenocarcinoma, is complementary and more sensitive and specific than thyroid transcription factor 1 in the differential diagnosis of primary pulmonary carcinoma: evaluation of 1674 cases by tissue microarray.

Cite this article as: Wang B, Jiang Y, Li SY, Niu RL, Blasberg JD, Kaifi JT, Liu G, Wang ZL. Breast metastases from primary lung cancer: a retrospective case series on clinical, ultrasonographic, and immunohistochemical features. Transl Lung Cancer Res 2021;10(7):3226-3235. doi: 10.21037/ tlcr-21-542
Arch Pathol Lab Med 2012;136:163-71.

28. Enrico D, Saucedo S, Bravo I. Breast metastasis from primary lung adenocarcinoma in a young woman: A case report and literature review. World J Clin Oncol 2019;10:269-78.

29. Mirrielees JA, Kapur JH, Szalkucki LM, et al. Metastasis of primary lung carcinoma to the breast: a systematic review of the literature. J Surg Res 2014;188:419-31.

30. Liu S, Li S, Hai J, et al. Targeting HER2 Aberrations in Non-Small Cell Lung Cancer with Osimertinib. Clin Cancer Res 2018;24:2594-604.

31. Mazières J, Peters S, Lepage B, et al. Lung cancer that harbors an HER2 mutation: epidemiologic characteristics and therapeutic perspectives. J Clin Oncol 2013;31:1997-2003.

32. Provenzano E, Byrne DJ, Russell PA, et al. Differential expression of immunohistochemical markers in primary lung and breast cancers enriched for triple-negative tumours. Histopathology 2016;68:367-77.

33. Whithaus K, Fukuoka J, Prihoda TJ, et al. Evaluation of napsin A, cytokeratin 5/6, p63, and thyroid transcription factor 1 in adenocarcinoma versus squamous cell carcinoma of the lung. Arch Pathol Lab Med 2012;136:155-62.

34. Brunnström H, Johansson L, Jirström K, et al. Immunohistochemistry in the differential diagnostics of primary lung cancer: an investigation within the Southern Swedish Lung Cancer Study. Am J Clin Pathol 2013;140:37-46.

35. Thike AA, Cheok PY, Jara-Lazaro AR, et al. Triplenegative breast cancer: clinicopathological characteristics and relationship with basal-like breast cancer. Mod Pathol 2010;23:123-33.

36. Livasy CA, Karaca G, Nanda R, et al. Phenotypic evaluation of the basal-like subtype of invasive breast carcinoma. Mod Pathol 2006;19:264-71.

37. Rakha EA, El-Sayed ME, Green AR, et al. Breast carcinoma with basal differentiation: a proposal for pathology definition based on basal cytokeratin expression. Histopathology 2007;50:434-8. 